\title{
SHORT RP TACHYCARDIA, SUCCESFULLY MANAGED WITH RADIO FREQUENCY ABLATION
}

\author{
Ajaz Ahmad Lone, Nisar Ahmad Tramboo, Syed Bilal
}

Department of Cardiology, SKIMS

35-year female patient presented with recurrent palpitations. Her baseline ECG was normal but during the episode of palpitations it revealed short RP tachycardia. Intracardiac ECG revealed orthodromic reentrant tachycardia (ORT) due to concealed left lateral pathway (Figure 1). She was successfully treated by radio-frequency ablation (RFA) through trans-septal approach. Figure 2 shows RV pacing getting conducted over left lateral pathway to atria which disappears after ablation of the pathway.

Paroxysmal supraventricular tachycardias (PSVT) are common arrhythmias affecting all ages, patients experience frequent palpitations which may be self-limiting or need management in hospital settings. PSVTs are generally of two types. Those related to and arising within the AV node referred to as AV node reentry tachycardia (AVNRT) and the other type involves a manifest or concealed accessory pathway referred as atrioventricular reentry tachycardia (AVRT). When the antegrade limb of the reentry circuit involves the AV node it presents as a narrow complex tachycardia also called as ORT. Antidromic tachycardia presents as wide QRS tachycardia as the antegrade limb involves the accessory pathway. Typical AVNRT and ORT both present with short RP tachycardia and acute management involves vagal maneuver like Valsalva maneuver and carotid sinus massage. IV medications like adenosine and verapamil or diltiazem frequently terminate this tachycardia. RFA is curative in such arrhythmias with high success rates exceeding $98 \%$ and low complication

\begin{tabular}{ll|l} 
& Access this article online \\
\hline & Website: \\
\hline
\end{tabular}

rates of less than $1 \%$. RFA is indicated in patients presenting with recurrent arrhythmias failing conservative approach or medical treatment.

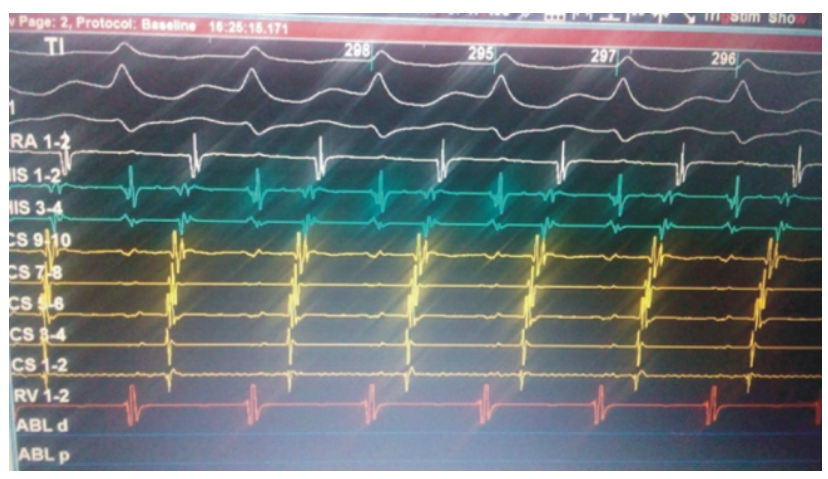

Fig. 1. Intracardiac ECG showing short RP tachycardia

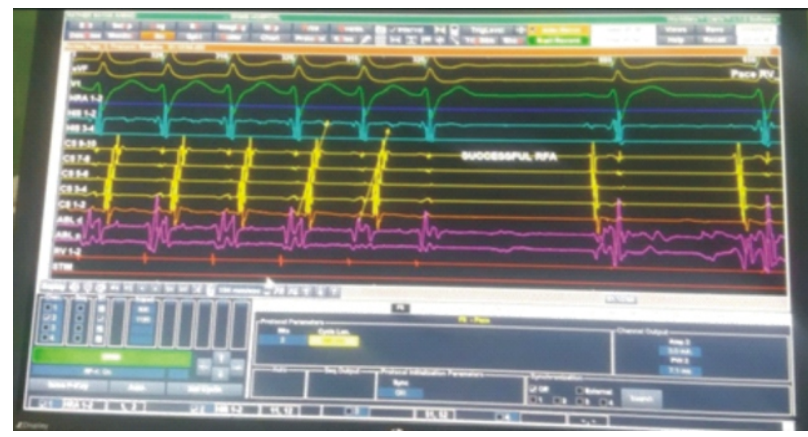

Fig. 2. RV pacing conducted over left lateral pathway to atria, disappears after ablation of the pathway.

Correspondence:

Dr. AjazAhmad Lone, DM

Additional Professor, Department of Cardiology, SKIMS

Email: drajaz666@gmail.com

How to cite this article: Lone A, Tramboo NA, Bilal S. SHORT RP TACHYCARDIA, SUCCESFULLY MANAGED WITH RADIO FREQUENCY ABLATION. JMS 2020; 23(3):36-37

Received: 2020-08-24 Accepted: 2020-08-29 
Lone A et al, Short RP Tachycardia, Succesfully Managed with Radio Frequency Ablation.
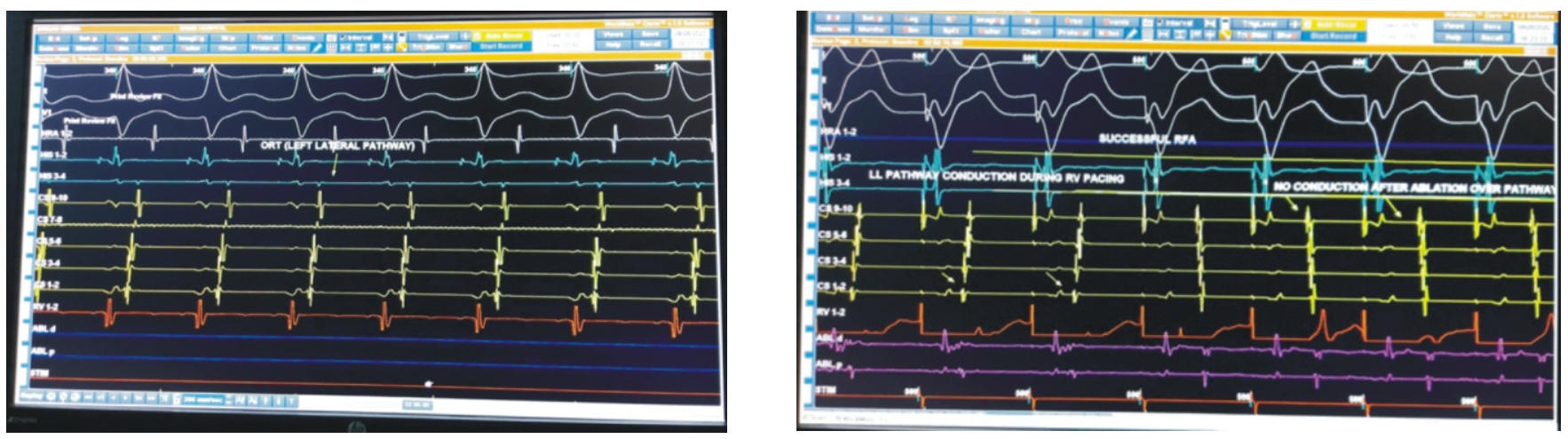